\title{
Análisis psicométrico del cuestionario de conductas antisociales en la infancia y adolescencia (CASIA) aplicado a población colombiana adolescente escolarizada*
}

\section{Psychometric analysis of the questionnaire of antisocial behavior in childhood and adolescence (CASIA) applied to Colombian adolescent schooled population}

\footnotetext{
Sergio Fabián Calderón ORCID: 0000-0003-4843-5647

Javier Andrés Santana

ORCID: 0000-0002-7993-1208

Fernando Riveros Munévar** ORCID: 0000-0002-0414-674X

Juan Carlos Borbón

ORCID: 0000-0001-7139-6346

Resumen

El objetivo de la presente investigación cuantitativa no experimental y de tipo psicométrico fue analizar las propiedades psicométricas del CASIA aplicado a población colombiana adolescente escolarizada de grados séptimo, octavo, noveno y décimo. El estudio se realizó con una muestra de 356 adolescentes con edades comprendidas entre los 11-18 años, pertenecientes a 4 centros educativos de educación básica y media, ubicados en la ciudad de Bogotá y Mosquera. Los resultados mostraron 6 factores, que explicaron el $51,51 \%$ de la varianza total acumulada, se presentó un

Corporación Universitaria Iberoamericana, Colombia

Recibido: 27 de julio de 2017 Revisado: 1 de septiembre de 2017 Aceptado: 20 de enero de 2018 alfa de Cronbach de, 755 y un coeficiente dos mitades de Guttman de, 749, indicando una escala confiable y consistente para población colombiana, con una estructura factorial clara y ajustada, que difiere con los estudios originales pero que muestra congruencia teórica con el constructo.

Palabras clave: adolescentes, análisis psicométrico, validez, confiabilidad, conducta antisocial.

Artículo de investigación. Como citar: Calderon, S. F., Santana, J. A., Riveros, M. F., \& Borbón, J. C. (2019). Análisis psicométrico del cuestionario de conductas antisociales en la infancia y adolescencia (CASIA) aplicado a población colombiana adolescente escolarizada. Diversitas: Perspectivas en Psicología, 15(1), 157-168. https://doi.org/10.15332/s1794-9998.2019.0001.12

Dirección postal: Corporación Universitaria Iberoamericana, Dirección Calle 67 \# 5-27, Bogotá, Colombia. CP 110231. Correspondencia: efriveros45@hotmail.com
} 


\section{Abstract}

The objective of this cross-sectional study, quantitative and psychometric court, was to analyze the psychometric properties of the CASIA in Colombian adolescent population schooled of seventh, eighth, ninth and tenth. The study was conducted with a sample of 356 adolescents whose ages were between 11-18 years belonging to 4 schools in primary and secondary education, located in the city of Bogota and Mosquera. The results showed six factors that explained the $51.51 \%$ of the total cumulative variance, a Cronbach's alpha of 755 and a coefficient of two halves of Guttman, 749 were obtained, indicating a reliable and consistent for the Colombian population level, with a structure set clear, which differs with the original studies but shows theoretical congruence with the construct.

Keywords: Adolescents, psychometric properties, validity, reliability, antisocial behavior.

\section{Introducción}

La adolescencia es entendida como el periodo de desarrollo del ser humano que se prolonga generalmente durante el rango entre los 11 a 20 años, en el cual, la madurez biológica y sexual son manifestadas y se pretende alcanzar la madurez emocional y social (Papalia, Olds, \& Feldman, 2009). Durante esta etapa del desarrollo aumenta la influencia de aspectos socio-ambientales sobre el comportamiento del adolescente (persuasión del grupo de pares, posición y estructura socio-económica, grado o nivel educativo, pautas de crianza, carente adaptación a los escenarios sociales próximos, entre otros). Estos aspectos podrían determinar la adquisición, desarrollo y mantenimiento de la conducta antisocial adolescente. De acuerdo con el escenario del sujeto y debido a la combinación de los factores mencionados, la consecuencia de tal conjunción podría influir en que los jóvenes expongan problemas de comportamiento, y que probablemente, lleguen a involucrarse en actos antisociales reflejados, en mayor o menor medida, en agresión (Erickson, 1992).

Ahora bien, teniendo en cuenta la particularidad de esta etapa y el afrontamiento del individuo en la adolescencia, una muestra considerable de adolescentes puede dar inicio de forma gradual a algún tipo de problema comportamental, que, de una u otra manera, tiene el riesgo de agravarse si no es abordado a tiempo (Saldaña, 2001). Un ejemplo de esto, es la presencia del comportamiento antisocial, que hace referencia a una serie de eventos o hechos que están desajustados con las normas sociales y/o legales causando daño y/o malestar al resto de la población (Romero, Sobral, \& Luengo, 1999).

Actualmente, existen múltiples términos para hacer referencia a la conducta antisocial, a saber, conductas agresivas e impulsivas y los trastornos o problemas de la conducta, entre otros. En general, el término conducta antisocial hace referencia a “(...) los diferentes comportamientos que reflejan trasgresión de las reglas sociales, que impliquen un conjunto de acciones o eventos contra los demás" (Kazdin \& Buela-Casal, 1996, p. 19).

De acuerdo con la clasificación que realizan Frías, López y Díaz (2003), se pueden encontrar escenarios en donde la conducta antisocial es valorada dentro de la categoría de violencia juvenil, delincuencia juvenil o trastorno disocial'. También cabe resaltar que la conducta antisocial no es puntual de un grupo etario. Por el contrario, lo que se hace necesario es tener como punto de partida la prolongación del comportamiento antisocial desde la infancia hasta la adolescencia y posteriormente, en la adultez (Justicia et al., 2006). La conducta antisocial hace referencia a una diversidad de actos que infringen violación de las normas sociales y los derechos de los demás; sin embargo, se deben tener en cuenta

1 Se realiza tal acotación con el fin de prever posibles disonancias entre términos que distorsionen el fin del estudio. 
las construcciones del contexto sociocultural en el que se gesta (Kazdin, 1988).

De la misma forma, Frías, López y Díaz (2003) consideran que los jóvenes, por aspectos inherentes a su naturaleza, supeditan en cierta medida su integración social, y por el contrario buscan independencia con el fin de hallar identidad. Los jóvenes son miembros que señalan las problemáticas de la sociedad; sin embargo, y aunque no legitimen las reglas en su totalidad, por lo general siguen las normas impuestas. La integración de los jóvenes en la sociedad girará en torno a las normas a las que ellos le otorguen legitimidad, y por ende se adherirán a espacios y condiciones particulares. Lo que es claro es el alto porcentaje de población adolescente participante en actos antisociales y delictivos y que es una problemática potencial para el óptimo desarrollo individual, social y económico de una nación (Morales, 2008; Organización Mundial de la Salud [OMS], 2003).

Los factores de riesgo asociados e identificados dentro de la magnitud del fenómeno, según Hawkins et al. (1998), hacen énfasis en que éstos determinan las condiciones del individuo o su ambiente, las cuales anticipan con mayor probabilidad de aparición y desarrollo del comportamiento violento, lo que en este caso puede considerarse un flagelo. Con base en la propuesta realizada por Correa, Manjarrés, Montes-Fontalbo y Polo-Suarez (2003), se concluye que los factores familiares que están relacionados con la violencia juvenil, son aquellas variables inmersas en el eje familiar. Es quizá, la posición desde donde el joven inicia socialmente su experiencia de vida, su estatus socioeconómico y sus oportunidades educativas, relacionado en gran medida con el ajuste de diversas áreas, a saber, la social, la psicológica y la cultural. Estudios muestran también que los patrones de personalidad están asociados también a la conducta antisocial (Morán, Carmona, \& Fínez, 2016).

En ese orden, Quiroga y Cryan (2005) señalan que parte de los fenómenos psicopatológicos en la adultez obedecen a factores relacionados con la funcionalidad de las experiencias familiares durante la niñez. De igual forma, Stone (1993, citado por Quiroga \& Cryan, 2005) menciona la importancia de experiencias traumáticas en la etapa infantil, tales como el abuso físico y sexual infantil, la carencia e inestabilidad de figuras parentales y reiteradas pérdidas de contexto, y la conexión con la manifestación de conductas antisociales. Los jóvenes que presentan comportamientos antisociales y delictivos en edades tempranas y que se perpetúan en el tiempo (niños pequeños y/o preadolescentes), conforman un grupo en alto riesgo para continuar con las mismas o similares conductas y, progresivamente, de mayor gravedad en la adultez (Gendreau, Little, \& Goggin, 1996).

Un estudio llevado a cabo por Sobral et al. (2000) en Santiago de Compostela en el cual se tienen en cuenta los posibles factores que afectan o generan la aparición de conductas antisociales, halló que dichas conductas tienen poca relación con el nivel socioeconómico de los estudiantes evaluados, contrario a la alta relación encontrada con los factores sociales, la poca filiación parental, el trato con otros jóvenes delincuentes y los lazos afectivos creados en el contexto escolar. Cabe tener en cuenta en los resultados arrojados por la investigación de Sobral et al. (2000), los hombres evaluados fueron quienes más reflejaron respuestas antisociales en comparación con las mujeres quienes solamente evidenciaron un bajo nivel de empatía. Estudios recientes llevados a cabo en Perú (Almonacid \& Rosario, 2017; Reyes, Noé, \& Gonzales, 2016) reafirman al clima familiar como una variable relacionada de forma importante con la conducta antisocial.

Finalmente, se ha mostrado que los comportamientos antisociales ampliamente conceptuados se relacionan con otros desórdenes, a saber, ansiedad, depresión y déficit de atención e hiperactividad (Angold et al., 1999; Maughan et al., 2004). De la misma forma, es importante explorar más adelante el papel que tienen los factores genéticos y ambientales en la explicación de la relación entre ES (Estimulo social) y el comportamiento antisocial. Esto, teniendo en cuenta que Tuvblad, Grann \& Lichtenstein (2006) mostraron que la heredabilidad del comportamiento antisocial varía en función del ambiente, muestran así las interacciones entre el genotipo transportador de serotonina de una familia de estratos bajos y la predicción de la delincuencia adolescente (Aslund et al., 2013). 
El estudio mencionado finaliza planteando la necesidad de una recopilación más exhaustiva de información entorno a la interacción de factores biológicos y ambientales para entender el papel del nivel socioeconómico en el desarrollo del comportamiento antisocial (Koenen, Uddin, Amstadter, \& Galea, 2010; Piotrowska, Stride, Croft, \& Rowe, 2015; Raine, 2002).

Otras investigaciones que resaltan la importancia del estudio de la conducta antisocial y delictiva muestran que el $16,6 \%$ de los adolescentes tenían un nivel alto de conductas antisociales, el $10 \%$ se inscribe en el perfil de alto riesgo y el 6,6\% en el perfil antisocial (Garaigordobil \& Maganto, 2016), o que los adolescentes con alta conducta antisocial se implican más en situaciones de bullying y cyberbullying en todos sus roles (víctimas, agresores y observadores) y usan significativamente más estrategias agresivas como estrategia para resolver conflictos (Garaigordobil, 2017).

Enfatizado en el contexto colombiano, se encuentra la investigación realizada por Sanabria y Uribe (2010) en la ciudad de Cali, en donde se estudiaron las manifestaciones de la conducta antisocial y delictiva en dos grupos de adolescentes (hombres y mujeres), jóvenes (entre los 12 y los 18 años de edad), que fueron clasificados como infractores de ley y no infractores. Los resultados refieren que 3.677 jóvenes presentaron algún tipo de conducta delictiva, siendo el hurto el acto delictivo más prevalente en ambos sexos.

Asimismo, un estudio realizado por Rodríguez, Espinosa \& Pardo (2013) en Ibagué (Colombia), indicó que el $84 \%$ de los adolescentes habían manifestado conductas de tipo antisocial y el $12 \%$ restante conductas delictivas, concluyeron que, si la familia no servía de grupo nuclear formativo en la adquisición de habilidades sociales para el establecimiento de vínculos sociales, se debía delegar esa función al ente de educación, el cual debía brindar espacios de socialización para la promoción del apego, las creencias en los lineamientos tradicionales, el carácter de compromiso y la actuación participativa.

Por su parte, Uribe, Sanabria, Orcasita, \& Barreto (2016) realizaron un estudio en cinco ciudades colombianas, y encontraron que las conductas anti- sociales se presentaban entre el $13 \%$ y el $40 \%$ de los adolescentes colombianos, las delictivas entre el $4 \%$ y el $15 \%$ y que existía una mayor prevalencia de ambas conductas en varones. Cabe resaltar que, a pesar de las altas cifras halladas, son escasas las investigaciones realizadas en Colombia con respecto a la representación de la conducta antisocial en la adolescencia, probablemente por instrumentos no válidos y confiables para el país, encontrándose sin embargo en dichos estudios la utilización del Cuestionario de Conductas Antisociales en la Infancia y Adolescencia (CASIA).

Dicho cuestionario fue elaborado por María Teresa Gonzales Martínez en el año 2012. Este instrumento de evaluación clínica para población infantil y adolescente (entre 8 y 15 años de edad), buscó identificar la aparición de conductas antisociales que se podían relacionar con trastornos de la personalidad.

A pesar del uso de la escala, estudios sobre sus propiedades psicométricas son escasos. Se resalta entonces, la investigación que realizó González (2012) -autora del mismo-, en España, con una muestra de 504 sujetos de edades entre los 8 a 15 años, de los cuales el $55 \%$ pertenecían a un medio urbano y el $45 \%$ a un medio rural, quien obtuvo una confiabilidad de 0,81. Del mismo modo, utilizó la validez de criterio externo cuyos resultados fueron satisfactorios y significativos a un nivel de confianza del 0,01 . La conclusión acerca del instrumento, según Gonzales (2012), es que presentó niveles altos de confiabilidad y validez. En Colombia suele utilizarse como escala de evaluación sin conocer sus datos de validez y confiabilidad, por tal razón, esta investigación tuvo como objetivo analizar las propiedades psicométricas del Cuestionario de Conductas Antisociales en la Infancia y Adolescencia (CASIA) aplicado a población colombiana adolescente escolarizada.

\section{Método}

\section{Tipo de estudio}

Se planteó un estudio instrumental, de acuerdo con la categorización de Montero \& León (2007), por cuanto está encaminado al análisis de las cualidades psicométricas de una prueba. 


\section{Participantes}

Se evaluaron 356 niños, niñas y adolescentes escolarizados, siendo 183 hombres $(51,4 \%$ ) y 173 mujeres $(48,6 \%)$, con edades comprendidas entre los $11 \mathrm{y}$ los 18 años ( $M=14,03$ años; $D E=1,315$ años), pertenecientes a 4 colegios colombianos $(2$ colegios rurales del municipio de Mosquera, Cundinamarca, siendo uno una institución pública y otra privada, y 2 colegios de la ciudad de Bogotá, siendo uno público y uno privado). La muestra fueobtenida por medio de un muestreo no probabilístico por sujetos disponibles. Los participantes se encontraban cursando los grados séptimo $(7 \mathrm{mo})$ a décimo $(10 \mathrm{mo})$ de formación básica media regular (tabla 1).

Tabla 1.

Distribución de los participantes según el grado académico en curso.

\begin{tabular}{ccc}
\hline Grado & Frecuencia & Porcentaje \\
\hline 7 & 81 & 22,8 \\
8 & 96 & 27,0 \\
9 & 113 & 31,7 \\
10 & 66 & 18,5 \\
Total & 356 & 100,0 \\
\hline
\end{tabular}

Fuente: elaboración propia.

\section{Instrumento}

El Cuestionario de Conductas Antisociales en la Infancia y Adolescencia (CASIA) permite obtener información objetiva sobre la conducta antisocial y sobre la conducta agresiva de un sujeto. Este cuestionario supone una aportación importante y de interés para el campo de la investigación y evaluación clínica infantil y del adolescente. Dicha escala en su estudio original presentó un criterio de confiabilidad de 0,812 (González, 2012).

La forma de aplicación del cuestionario puede ser individual y/o colectiva, con un tiempo de duración de doce minutos aproximadamente. Consta de 20 reactivos en escala puntuación de 0 a 2 , siendo 0 "nunca", 1 siendo "algunas veces" y 2 siendo "siempre", que se distribuyen de la siguiente manera: los ítems $1,4,5,6,7,10,11,15,19$ y 20 se relacionan con la aparición de conductas antisociales con agresividad, y los ítems $2,3,8,9,12,13,14,16,17$, y 18 se relacionan con la aparición de conductas antisociales sin agresividad.

\section{Procedimiento}

Inicialmente se realizó la invitación formal a las instituciones educativas que desearon participar en la aplicación del instrumento y recibiendo respuesta positiva de cuatro instituciones. De acuerdo con la cantidad de estudiantes referidos por los rectores y/o coordinadores de las instituciones participantes, se hizo el envío de los consentimientos a los padres de familia de cada alumno. Posteriormente se programó la fecha y espacio para la aplicación de la prueba donde los participantes firmaron el asentimiento informado. En total, se proyectó la participación de 420 estudiantes, de los cuales 356 aceptaron su participación de acuerdo con la firma del consentimiento y asentimiento correspondientes.

Al momento de ejecutar la prueba se trasladaron los estudiantes a un aula dispuesta por la institución y en donde se les explicó la confidencialidad del ejercicio, el tiempo de respuesta y las instrucciones pertinentes. Cabe resaltar que, tanto la ejecución, medición, y tiempo de aplicación de instrumento se realizaron bajo la normatividad establecida de la prueba con el fin de mantener el criterio original de evaluación.

De igual manera se estableció con los colegios un reporte de los resultados generales de la aplicación, salvaguardando y respetando el criterio de confidencialidad de cada prueba. Lo anterior con el fin de que las instituciones intervinieran de manera adecuada las posibles conductas evidenciadas en los resultados.

Posterior a la aplicación de la prueba CASIA, se realizaron los análisis de los datos y la elaboración del documento final. Los datos fueron analizados mediante el paquete estadístico SPSS, versión 20. Se tomó el criterio de confiabilidad con valores por encima de 0,7, para datos de adecuación muestral superiores a 0,7 y de significancia menores a 0,05, extracción de reactivos mayores a 0,3 y factores 
obtenidos con autovalores superiores a 1 , número de factores que se confirmaron con el método paralelo de Horn por medio del programa R.

\section{Resultados}

Los análisis de resultados se mostrarán en dos apartados, siendo el primero el análisis de validez de constructo desde el análisis factorial exploratorio, y un segundo apartado sobre las condiciones de confiabilidad y consistencia interna de la prueba.

\section{Análisis factorial exploratorio}

Con el fin de determinar si el tamaño muestral fue acorde para realizar los análisis factoriales, se procedió a ejecutar las pruebas de adecuación muestral de Kaiser-Meyer-Olkin y la prueba de esfericidad de Bartlett (tabla 2).

Tabla 2. Pruebas de adecuación muestral.

\begin{tabular}{lll}
\hline $\begin{array}{l}\text { Pruebas de Kaiser-Meyer- } \\
\text { Olkin y Bartlett }\end{array}$ & \\
\hline $\begin{array}{l}\text { Medida de adecuación } \\
\text { muestral de Kaiser-Me- }\end{array}$ & 0,736 & \\
yer-Olkin. & & \\
\hline $\begin{array}{l}\text { Prueba de esfericidad de } \\
\text { Bartlett }\end{array}$ & $\begin{array}{l}\text { Chi-cuadrado } \\
\text { aproximado }\end{array}$ & 1069,208 \\
\hline & gl & 190 \\
\hline & Sig. & 0,000 \\
\hline
\end{tabular}

Fuente: elaboración propia.

Los resultados de la tabla 2 muestran un KMO superior a 0,7 y una significancia en la prueba de esfericidad de Bartlett inferior a 0,05, lo que refiere un tamaño muestral apropiado para realizar el análisis factorial, el cual se realizó por medio de la extracción de componentes principales con rotación Varimax (dicho método de rotación fue elegido debido a las ortogonalidades de la matriz).
En la tabla 3 se muestran los niveles de extracción de cada reactivo.

Tabla 3. Extracción de cada reactivo del CASIA.

\begin{tabular}{cc}
\hline Reactivo & Extracción \\
\hline Item1 & 0,756 \\
Item2 & 0,413 \\
Item3 & 0,570 \\
Item4 & 0,733 \\
Item5 & 0,691 \\
Item6 & 0,593 \\
Item7 & 0,559 \\
Item8 & 0,620 \\
Item9 & 0,588 \\
Item10 & 0,742 \\
Item11 & 0,689 \\
Item12 & 0,606 \\
Item13 & 0,509 \\
Item14 & 0,629 \\
Item15 & 0,684 \\
Item16 & 0,563 \\
Item17 & 0,610 \\
Item18 & 0,667 \\
Item19 & 0,588 \\
Item20 & 0,571 \\
\hline
\end{tabular}

Fuente: elaboración propia.

Dados los resultados de la tabla 3 , se encuentra que la totalidad de reactivos superó el valor de 0,3 , el cual fue el valor mínimo establecido para conservar los ítems, lo que se recomendó incluirlos en su totalidad. Tras esto se obtuvieron los datos de los factores obtenidos y la varianza total explicada (tabla 4). 
Tabla 4. Componentes extraídos y varianza explicada.

\begin{tabular}{cccc}
\hline Componente & Total & $\begin{array}{c}\text { \% de la } \\
\text { varianza }\end{array}$ & \% acumulado \\
\hline 1 & 1,701 & 8,504 & 8,504 \\
2 & 1,666 & 8,331 & 16,835 \\
3 & 1,637 & 8,185 & 25,019 \\
4 & 1,619 & 8,096 & 33,116 \\
5 & 1,559 & 7,794 & 40,910 \\
6 & 1,553 & 7,763 & 48,673 \\
7 & 1,466 & 7,330 & 56,003 \\
8 & 1,181 & 5,903 & 61,906 \\
\hline
\end{tabular}

Fuente: elaboración propia.

Los resultados de la tabla anterior muestran que se obtuvieron 8 componentes extraídos, que explicaron el $61,91 \%$ de la varianza obtenida. Posteriormente se realizó la matriz de componentes rotados (tabla 5) para verificar la agrupación de los reactivos a la luz de los componentes.

Tabla 5. Matriz de componentes rotados.

\begin{tabular}{|c|c|c|c|c|c|c|c|c|}
\hline & 1 & 2 & 3 & 4 & 5 & 6 & 7 & 8 \\
\hline Item11 & 0,777 & & & & & & & \\
\hline Item14 & 0,685 & & & & & & & \\
\hline Item16 & 0,452 & & 0,397 & & & & & \\
\hline Item2 & & 0,460 & & & & & & \\
\hline Item10 & & 0,731 & & & & & & \\
\hline Item19 & & 0,713 & & & & & & \\
\hline Item4 & & & 0,229 & & & & & \\
\hline Item8 & & & 0,703 & & & & & \\
\hline Item15 & & & 0,444 & & 0,513 & & & \\
\hline Item17 & & & 0,727 & & & & & \\
\hline Item5 & & & & 0,808 & & & & \\
\hline Item6 & & & & 0,494 & & & & \\
\hline Item9 & & & & 0,266 & & & & \\
\hline
\end{tabular}

\begin{tabular}{|c|c|c|c|c|c|c|c|}
\hline & 1 & 2 & 3 & 4 & 5 & 6 & 7 \\
\hline Item12 & & & & 0,669 & & & \\
\hline Item7 & & & & & 0,672 & & \\
\hline Item13 & & & & & 0,635 & & \\
\hline Item18 & & & & & 0,216 & & \\
\hline Item1 & & & & & & 0,825 & \\
\hline Item3 & & & & & & 0,626 & \\
\hline Item20 & & & & & & 0,446 & \\
\hline
\end{tabular}

Fuente: elaboración propia.

La matriz de la tabla 5 muestra que de los 8 componentes obtenidos, 2 de ellos no presentaron carga de reactivos, por lo cual, realmente se obtuvieron 6 componentes (número de factores confirmados por medio del análisis paralelo de Horn), procediendo a realizar nuevamente el análisis factorial ajustado a 6 factores fijos (tabla 6).

Tabla 6. Componentes extraídos y varianza explicada, ajustada a 6 factores.

\begin{tabular}{cccc}
\hline Componente & Total & $\begin{array}{c}\text { \% de la } \\
\text { varianza }\end{array}$ & $\begin{array}{c}\% \\
\text { acumulado }\end{array}$ \\
\hline 1 & 1,891 & 9,453 & 9,453 \\
2 & 1,814 & 9,069 & 18,522 \\
3 & 1,807 & 9,035 & 27,557 \\
4 & 1,674 & 8,368 & 35,925 \\
5 & 1,625 & 8,127 & 44,052 \\
6 & 1,492 & 7,461 & 51,513 \\
\hline
\end{tabular}

Fuente: elaboración propia.

La matriz ajustada mostró que dichos 6 factores explicaban el $51,513 \%$ de la varianza total acumulada, y la matriz de componentes rotados ajustados (tabla 7) mostraron mejor ajuste de los reactivos, con claridad sobre las cargas de los mismos en sus factores. 
Tabla 7. Matriz de componentes rotados, ajustada a 6 componentes.

\begin{tabular}{|c|c|c|c|c|c|c|}
\hline & Compo & nnente & & & & \\
\hline & 1 & 2 & 3 & 4 & 5 & 6 \\
\hline Item9 & 0,646 & & & & & \\
\hline Item14 & 0,609 & & & & & \\
\hline Item18 & 0,553 & & & & & \\
\hline Item4 & & 0,548 & & & & \\
\hline Item8 & & 0,691 & & & & \\
\hline Item16 & & 0,528 & & & & \\
\hline Item17 & & 0,705 & & & & \\
\hline Item7 & & & 0,648 & & & \\
\hline Item11 & & & 0,516 & & & \\
\hline Item13 & & & 0,622 & & & \\
\hline Item2 & & & & 0,493 & & \\
\hline Item10 & & & & 0,736 & & \\
\hline Item19 & & & & 0,664 & & \\
\hline Item5 & & & & & 0,782 & \\
\hline Item6 & & & & & 0,441 & \\
\hline Item12 & & & & & 0,695 & \\
\hline Item1 & & & & & & 0,708 \\
\hline Item3 & & & & & & 0,483 \\
\hline Item15 & & & & & & 0,316 \\
\hline Item20 & & & & & & 0,484 \\
\hline
\end{tabular}

Fuente: elaboración propia.

\section{Consistencia interna y confiabilidad del cuestionario}

Con el fin de establecer los datos de consistencia interna de la prueba, se procedió a obtener el alfa de Cronbach, evidenciando un coeficiente de 0,755. La tabla 8 muestra las correlaciones entre los elementos y el total de la prueba, así como los cambios en el alfa de Cronbach si se eliminara el elemento, mostrando que todos los reactivos arrojaron correlaciones positivas frente al total, y alfas afectados negativamente en caso hipotético de eliminar el reactivo, o con cambios pequeños en los mismos.
Tabla 8. Correlaciones entre elemento-total de la prueba y Alfa si se elimina el reactivo.

\begin{tabular}{lcc}
\hline & $\begin{array}{c}\text { Correlación } \\
\text { elemento-total } \\
\text { corregida }\end{array}$ & $\begin{array}{c}\text { Alfa de Cronbach si se } \\
\text { elimina el elemento }\end{array}$ \\
\hline Item1 & 0,296 & 0,748 \\
Item2 & 0,294 & 0,747 \\
Item3 & 0,378 & 0,741 \\
Item4 & 0,237 & 0,751 \\
Item5 & 0,157 & 0,754 \\
Item6 & 0,417 & 0,738 \\
Item7 & 0,169 & 0,754 \\
Item8 & 0,217 & 0,752 \\
Item9 & 0,343 & 0,743 \\
Item10 & 0,242 & 0,750 \\
Item11 & 0,322 & 0,745 \\
Item12 & 0,387 & 0,741 \\
Item13 & 0,302 & 0,746 \\
Item14 & 0,455 & 0,733 \\
Item15 & 0,312 & 0,746 \\
Item16 & 0,421 & 0,736 \\
Item17 & 0,302 & 0,747 \\
Item18 & 0,291 & 0,747 \\
Item19 & 0,280 & 0,748 \\
Item20 & 0,469 & 0,731 \\
\hline & &
\end{tabular}

Fuente: elaboración propia.

Finalmente, para determinar los datos de confiabilidad de la escala, se realizó la prueba de división por mitades de Guttman (tabla 9). Los datos de confiabilidad por dos mitades mostraron coeficientes Alfa de Cronbach para la primera mitad de 0,525 y 0,666 para la segunda parte de la prueba, con correlaciones entre ella de 0,609 y un coeficiente final de Guttman de 0,749. 
Tabla 9. Estadísticos de confiabilidad por dos mitades.

\begin{tabular}{|c|c|c|c|}
\hline \multicolumn{4}{|c|}{ Confiabilidad por dos mitades } \\
\hline \multirow[t]{5}{*}{ Alfa de Cronbach } & \multirow[t]{2}{*}{ Parte 1} & Valor & 0,525 \\
\hline & & $\mathrm{N}$ de elementos & $10 a$ \\
\hline & \multirow[t]{2}{*}{ Parte 2} & Valor & 0,666 \\
\hline & & $\mathrm{N}$ de elementos & $10 \mathrm{~b}$ \\
\hline & \multicolumn{2}{|c|}{$\mathrm{N}$ total de elementos } & 20 \\
\hline \multicolumn{3}{|c|}{ Correlación entre formas } & 0,609 \\
\hline \multirow{2}{*}{$\begin{array}{l}\text { Coeficiente de } \\
\text { Spearman-Brown }\end{array}$} & \multicolumn{2}{|c|}{ Longitud igual } & 0,757 \\
\hline & \multicolumn{2}{|c|}{ Longitud desigual } & 0,757 \\
\hline \multicolumn{3}{|c|}{ Dos mitades de Guttman } & 0,749 \\
\hline \multicolumn{4}{|c|}{$\begin{array}{l}\text { a. Los elementos son: Item1, Item2, Item3, Item4, } \\
\text { Item5, Item6, Item7, Item8, Item9, Item10. } \\
\text { b. Los elementos son: Item11, Item12, Item13, } \\
\text { Item14, Item15, Item16, Item17, Item18, Item19, } \\
\text { Item20. }\end{array}$} \\
\hline
\end{tabular}

Fuente: elaboración propia.

\section{Discusión}

Es preciso mencionar que existen una multiplicidad de factores respecto a la condición antisocial. Estudios realizados demuestran la alta comorbilidad de trastornos asociados al desarrollo biológico y social de los adolescentes y la probabilidad de caer en conductas delictivas (Gendreau et al., 1996). Teniendo en cuenta que la manifestación de conductas antisociales en los niños, niñas y adolescentes, parte de una transgresión de las normas sociales y de la violación de los derechos de los demás, con una importante significación social, puesto que repercuten los efectos de manera negativa en el contexto social y familiar del sujeto, el abordaje en términos de evaluación e intervención demanda la actuación de diferentes áreas de la Psicología: clínica infantil, educativa, social, entre otras. Tal y como menciona Gonzales (2012), la sociedad actual se ha visto inmersa en diversos tipos de comportamiento que traen consigo consecuencias negativas tanto en el ámbito social y familiar del individuo. Dicho así, los procesos evaluativos y de intervención en esta población, requieren del uso y disposición de instrumentos de evaluación útiles y objetivos que permitan cuantificar estas conductas, objetivo al que se intentó aportar en este estudio.

En este orden de ideas, los resultados de la presente investigación mostraron desde el análisis factorial que la totalidad de los reactivos son apropiados para medir el constructo ya nombrado, mostrando además 6 factores claramente constituidos que explicaron el $51,51 \%$ de la varianza total acumulada. Los factores obtenidos difieren parcialmente de la versión original y del manual de la prueba del Cuestionario de Conductas Antisociales en la Infancia y Adolescencia (CASIA) (Gonzales, 2012), en tanto que en ellos hallaron 8 factores. Sin embargo, la diferencia radica en que un factor obtenido en el presente estudio recogió los reactivos de dos factores, fenómeno que ocurrió dos veces (una vez por cada categoría independiente, lo que explica el pasar de 8 a 6 factores), pero que al revisarlo semánticamente guardaban coherencia y conservaban las dos categorías no independientes, ya que ambas son consideradas componentes del constructo de conducta antisocial: 10 ítems se refieren a conductas en las que existe un componente de agresividad, y los otros 10 ítems, hacen referencia a conductas cuya característica específica es que transgreden el orden social, y las normas sociales, éticas y morales que regulan el comportamiento interactivo con los demás. Lo anterior, a pesar de la discrepancia de 8 a 6 factores, muestra la coherencia entre los reactivos y su organización factorial acorde, lo que permite afirmar la validez de la escala para medir la conducta antisocial.

Por otro lado, los datos de confiabilidad (alfa de Cronbach $=0,755$ ) y consistencia interna (dos mitades de Guttman $=0,749$ ) muestran una escala consistente en sí misma y con datos apropiados de confiabilidad para la población escolarizada objeto de investigación. Estos datos de confiabilidad son similares a los hallados en la versión original del Cuestionario de Conductas Antisociales en la Infancia y Adolescencia (CASIA) (Gonzales, 2012), donde se determinó la fiabilidad a través de la consistencia interna del mismo, mediante un alfa de Cronbach $=0,812$.

Respecto a las limitaciones que tuvo el estudio se puede concluir que, aun cuando el tamaño muestral era apropiado (Lloret-Segura, Ferreres-Traver, 
Hernández-Baeza, \& Tomás-Marco, 2014) y la matriz de correlaciones mostraba un adecuado ajuste para los análisis factoriales ( $\mathrm{KMO}=0,736$; Bartlett sig. $=0,000$ ), la muestra puede contar con sesgos, tanto por ser una muestra con alta o baja conducta antisocial, lo cual sumado a que el muestreo fue no probabilístico y pertenecientes a dos municipios de una misma región, aumenta la probabilidad de observar dichos sesgos. Por esto se recomiendan futuros estudios con muestras más amplias y de diferentes lugares del país, para garantizar la representatividad de la población. Otra limitante radica en que no se realizó una prueba piloto, que hubiese permitido aclarar términos que probablemente no se ajustan a la población colombiana, ya que la prueba es originaria de España lo cual permite recomendar para otra fase de esta línea de investigación la necesidad de iniciar por adaptaciones del lenguaje de la prueba.

Frente a posibles investigaciones futuras, se recomienda tener en cuenta aspectos de adaptación cultural de la prueba, especialmente en términos no apropiados para Colombia, estudios de validez de criterio (comparando resultados de esta prueba y el Cuestionario A-D de conductas antisociales y delictivas de Seisdedos, o comparando puntajes de población escolarizada regular y personas que se encuentren bajo responsabilidad penal). Ya que, en las posibles aplicaciones del instrumento, se recomiendan estudios descriptivos acerca de la presencia de conductas antisociales en los mismos niños escolarizados, comparativos según regiones o lugares del país, estratos socioeconómicos, sexo, edad u otras variables de interés, e inclusive estudios transculturales que permitan comparar los comportamientos presentados en Colombia frente a otros países latinoamericanos o de otros continentes.

Finalmente, este estudio arroja datos preliminares de la validez y de la consistencia del Cuestionario de Conductas Antisociales en la Infancia y Adolescencia (CASIA) en población Colombiana adolescente escolarizada entre $7^{\circ}$ a $10^{\circ}$ grado. Con lo cual se espera que se transforme en un insumo importante para continuar en esta línea de investigación, tanto en la validez de la prueba como en la evaluación de conductas antisociales en esta población, de forma tal que puedan realizarse diagnósticos preventivos y procesos de intervención necesarios para la disminución de las mismas y un manejo acertado del comportamiento infantil y adolescente colombiano. Esto supondrá un aporte importante y de interés para el campo clínico e investigativo en materia de niñez y adolescencia, mediante la contribución a determinar los contenidos de aparición, desarrollo y mantenimiento de tales conductas con el fin del mejoramiento de la convivencia social e interacción interpersonal en las diferentes áreas de ajuste de niños, niñas y adolescentes.

\section{Referencias}

Almonacid, J. \& Rosario, F. (2017). Clima Social Familiar y Conductas Antisociales en adolescentes de nivel secundario del distrito de Puente Piedra, Lima 2017. (Tesis de pregrado). Universidad Cesar Vallejo. Recuperado de http://repositorio.ucv.edu.pe/bitstream/handle/UCV/3166/Almonacin_MJC. pdf?sequence $=1$ \&isAllowed $=y$

Angold, A., Costello, E.J., \& Erkanli, A. (1999). Comorbidity. Journal of Child psychology and Psychiatry, 40(1), 57-87. Doi: https://doi. org/10.1111/1469-7610.00424

Aslund, C., Comasco, E., Nordquist, N., Leppert, J., Oreland, L., \& Nilsson, K.W. (2013). Selfreported family socioeconomic status, the 5HTTLPR genotype, and delinquent behavior in a community-based adolescent population. Aggressive Behavior, 39(1), 52-63. Doi:https:// doi.org/10.1002/ab.21451

Correa, D. M., Manjarrés M., N. P., Montes Fontalvo, J., \& Polo Suárez, C. R. (2003). Factores familiares, educativos y políticos asociados a la violencia en jóvenes del sector urbano del municipio de Ciénaga (Magdalena). Psicología desde el Caribe, 11, 57-74.

Erickson, M. T. (1992). Behavior disorders of children and adolescents. New Jersey: Prentice Hall, Inc.

Frías, M., López, S., \& Díaz, G. (2003). Predictores de la conducta antisocial juvenil: un 
modelo ecológico. Estudios de Psicología, 8, 15-24. Doi: https://doi.org/10.1590/ S1413-294X2003000100003

Garaigordobil, M. (2017). Conducta antisocial: conexión con bullying/cyberbullying y estrategias de resolución de conflictos. Psychosocial Intervention, 26, 47-54. Doi: https://doi. org/10.1016/j.psi.2015.12.002

Garaigordobil, M. \& Maganto, C. (2016). Conducta antisocial en adolescentes y jóvenes: prevalencia en el País Vasco y diferencias en función de variables socio-demográficas. Acción psicológica, 13(2), 57-68. Doi: https://doi.org/10.5944/ ap.13.2.17826

Gendreau, P., Little, T., \& Goggin, C. (1996). A meta-analysis of the predictors of adult offender recidivism: What works? Criminology, 34, 575-606. Doi: https://doi. org/10.1111/j.1745-9125.1996.tb01220.x

González, M. (2012). Cuestionario de Conductas Antisociales (CASIA), Madrid, España: CEPE.

Hawkins, J.D., Herrenkohl, T., Farrington, D.P., Brewer, D., Catalano, R.F., \& Harachi, T.W. (1998). A review of predictors of youth violence. En R. Loeber y D. Farrington (Eds.) Serious 292 and violent juvenile offenders: Risk factors and successful interventions. Thousand Oaks, CA: Sage.

Justicia, F., Benítez, J. L., Pichardo, M., Fernández, E., García, T., \& Fernández, M. (2006). Aproximación a un nuevo modelo explicativo del comportamiento antisocial. Revista Electrónica de Investigación Psicoeducativa,4(2), 131-150.

Kazdin, A. E. (1988). Tratamiento de la conducta antisocial en la infancia y la adolescencia. Madrid: Martínez Roca.

Kazdin, A. E. \& Buela-Casal, G. (1996). Conducta antisocial evaluación, tratamiento y prevención en la infancia y adolescencia. Madrid: Ediciones Pirámide.
Koenen, K.C., Uddin, M., Amstadter, A. B., \& Galea, S. (2010). Incorporating the social environment in genotype environment interaction studies of mental disorders. International Journal of Clinical Practice, 64(11), 1489- 1492. Doi: https:// doi.org/10.1111/j.1742-1241.2010.02359.x

Lloret-Segura, S., Ferreres-Traver, A., HernándezBaeza, A., \& Tomás-Marco, I. (2014) El análisis factorial exploratorio de los ítems: una guía práctica, revisada y actualizada. Anales de Psicología, 30(3), 1151-1169. Doi: https://doi. org/10.6018/analesps.30.3.199361

Maughan, B., Rowe, R., Messer, J., Goodman, R., \& Meltzer, H. (2004). Conduct disorder and oppositional defiant disorder in a national sample: Developmental epidemiology. Journal of Child Psychology and Psychiatry, 45(3), 609-621. Doi: https://doi. org/10.1111/j.1469-7610.2004.00250.x

Montero, I. \& León, O. (2007). A guide for naming research studies in Psychology. International Journal of Clinical and Health Psychology, 7(3), 847-862.

Morales, H. (2008). Factores asociados y trayectorias del desarrollo del comportamiento antisocial durante la adolescencia: implicaciones para la prevención de la violencia juvenil en América Latina. Interamerican Journal of Psychology, 42, 129-142.

Morán, C. Carmona, J., \& Fínez, M. (2016). Tipos de personalidad, agresión y conducta antisocial en adolescentes. Psychology, Society, \& Education, 8(1), 65-80. Doi: https://doi. org/10.25115/psye.v8i1.548

Organización Mundial de la Salud. (2003). Informe mundial sobre la violencia y la salud. Washington: O.M.S.

Papalia, D., Olds, S., \& Feldman, R. (2009). Desarrollo Humano. De la infancia a la adolescencia. (11va. edición). México: Mc Graw Hill.

Piotrowska, P., Stride, C., Croft, S., \& Rowe, R. (2015). Socioeconomic status and antisocial 
behaviour among children and adolescents: $\mathrm{A}$ systematic review and meta-analysis. Clinical Psychology Review, 35, 47-55. Doi: https://doi. org/10.1016/j.cpr.2014.11.003

Quiroga, S. \& Cryan, G. (2005). Estudio sociodemográfico y epidemiológico de una población adolescentes en riesgo psicosocial. Memorias de las XII Jornadas de Investigación: "Avances, Nuevos Desarrollos e Integración Regional”. Buenos Aires: Universidad de Buenos Aires.

Raine, A. (2002). Biosocial studies of antisocial and violent behavior in children and adults: A review. Journal of Abnormal Child Psychology, 30(4), 311-326. Doi: https://doi. org/10.1023/A:1015754122318

Reyes, L., Noé, H., \& Gonzales, M. (2016). Conductas antisociales y clima social familiar en estudiantes de secundaria en instituciones educativas públicas de la ciudad de Huarmey. (Tesis de pregrado). Universidad Cesar Vallejo. Recuperado de http://repositorio.ucv.edu.pe/bitstream/handle/UCV/411/ reyes_hl.pdf?sequence $=1$ \&isAllowed $=y$

Rodríguez, H. Espinosa, A., \& Pardo, C. (2013). Función familiar y conductas antisociales y delictivas de adolescentes de instituciones públicas educativas de la ciudad de Ibagué Colombia. Vanguardia Psicológica, 3(2), 137-149.
Romero, E., Sobral, J., \& Luengo, M. A. (1999). Personalidad y Delincuencia: Entre la Biología y la Sociedad. Santiago de Compostela: Grupo Editorial Universitario.

Saldaña, C. (2001). Detección y prevención en el aula de los problemas del adolescente. Madrid: Pirámide.

Sanabria, A. \& Uribe, A. (2010). Factores psicosociales de riesgo asociados a conductas problemáticas en jóvenes infractores y no infractores. Diversitas: Perspectivas en Psicología, 6(2), 257-274. Doi: https://doi.org/10.15332/ s1794-9998.2010.0002.04

Sobral, J. Romero, E. Luengo, A., \& Marzoa, J. (2000). Personalidad y conducta antisocial: amplificadores individuales de los efectos contextuales. Psicothema, 12(4), 661-670.

Tuvblad, C., Grann, M., \& Lichtenstein, P. (2006). Heritability for adolescent antisocial behavior differs with socioeconomic status: Gene-environment interaction. Journal of Child Psychology and Psychiatry, 47(7), 734-743. Doi: https://doi. org/10.1111/j.1469-7610.2005.01552.x

Uribe, A.F., Sanabria, A. M., Orcasita, L., \& Castellanos, J. (2016). Conducta antisocial y delictiva en adolescentes y jóvenes colombianos. Informes Psicológicos, 16(2), 103-119. Doi: http://doi.org/10.18566/infpsicv16n2a07 